\title{
The CD4+ T-cell transcriptome and serum lgE in asthma: IL17RB and the role of sex
}

\author{
Gary M Hunninghake ${ }^{1,2^{*}}$, Jen-hwa Chu1', Sunita S Sharma ${ }^{1,2}$, Michael H Cho $0^{1,2}$, Blanca E Himes ${ }^{1,3,4}$, \\ Angela J Rogers ${ }^{1,2}$, Amy Murphy', Vincent J Carey ${ }^{1,3}$ and Benjamin A Raby ${ }^{1,2,3}$
}

\begin{abstract}
Background: The relationships between total serum lgE levels and gene expression patterns in peripheral blood CD4+ T cells (in all subjects and within each sex specifically) are not known.

Methods: Peripheral blood CD4+ T cells from 223 participants from the Childhood Asthma Management Program (CAMP) with simultaneous measurement of IgE. Total RNA was isolated, and expression profiles were generated with Illumina HumanRef8 v2 BeadChip arrays. Modeling of the relationship between genome-wide gene transcript levels and IgE levels was performed in all subjects, and stratified by sex.
\end{abstract}

Results: Among all subjects, significant evidence for association between gene transcript abundance and lgE was identified for a single gene, the interleukin 17 receptor B (IL17RB), explaining $12 \%$ of the variance $\left(r^{2}\right)$ in $\lg E$ measurement ( $p$ value $=7 \times 10^{-7}, 9 \times 10^{-3}$ after adjustment for multiple testing). Sex stratified analyses revealed that the correlation between IL 17RB and IgE was restricted to males only $\left(r^{2}=0.19, \mathrm{p}\right.$ value $=8 \times 10^{-8}$; test for sexinteraction $p<0.05$ ). Significant correlation between gene transcript abundance and IgE level was not found in females. Additionally we demonstrated substantial sex-specific differences in IgE when considering multi-gene models, and in canonical pathway analyses of IgE level.

Conclusions: Our results indicate that IL17RB may be the only gene expressed in CD4+ T cells whose transcript measurement is correlated with the variation in lgE level in asthmatics. These results provide further evidence sex may play a role in the genomic regulation of lgE.

\section{Background}

Total serum immunoglobulin E (IgE) is a risk factor for both the development of [1] and disease severity in asthma [2]. The production of IgE is controlled by a complex regulatory process that ultimately involves isotype class switching by mononuclear B lymphocytes, [3] a CD4+ T cell dependent process [3]. However, to our knowledge, there has been no analysis of the correlation between genome-wide CD4+ T cell gene expression and the variability in serum IgE among asthmatics.

Sex is a critical determinant of IgE level, with males having a stronger tendency towards higher total IgE than females [4]. Sex-related differences in IgE are present early in life [5] and persist into adulthood [6]. Furthermore, there is growing evidence that the

\footnotetext{
* Correspondence: ghunninghake@partners.org

'Channing Laboratory, Brigham and Women's Hospital, Harvard Medical School, Boston, MA 02115, USA

Full list of author information is available at the end of the article
}

underlying molecular underpinnings of IgE levels differ between males and females. For example, we have previously demonstrated striking differences by sex in IgE heritability estimates, with $83 \%$ of the variability in IgE levels attributable to genetic factors in males, as compared to $63 \%$ in females [7]. Moreover, evidence for sexually-distinct genetic determinants of IgE has been demonstrated in genome-wide linkage analyses of IgE in some, [7] but not all studies [8].

Based on these findings we hypothesized that genomewide CD4+ T cell microarray analyses would provide unique insights into genes that correlate with the variability in IgE level, and that sex would be an important modifier of these correlations. To test these hypotheses we explored the relationship between total serum IgE levels and genome-wide gene expression transcript abundance in a cohort of 223 young adults with asthma, both in all subjects and within each sex specifically.

\section{() Biomed Central}




\section{Methods}

\section{Study population and sample collection}

CAMP was a multicenter clinical trial of the effects of anti-inflammatory medications in children with mild to moderate asthma. All participants had asthma defined by symptoms greater than twice per week, use of an inhaled bronchodilator at least twice weekly or use of daily medication for asthma, and increased airway responsiveness [9]. Participant data relating to atopy was obtained from the original CAMP dataset. The completion of the trial was followed by two four-year observation studies - CAMP Continuation Study (CAMPCS) 1 and 2. During Years 3 and 4 of CAMPCS/2, 299 children at four of eight CAMP study centers (Baltimore, Boston, Denver, St. Louis) participated in an ancillary study of gene expression in asthma. The study visit included questionnaire assessments of asthma severity, measurements of pulmonary function, and collection of peripheral blood for the simultaneous measurement of cell count, total serum IgE, and CD4+ T cell gene expression. Samples for RNA extraction were collected with BD Vacutainer CPT tubes, (BD Diagnostics, Franklin Lakes, New Jersey) placed on ice, centrifuged within 1 hour (20 minutes at 1700RCF) followed by mononuclear cell layer isolation and suspension in $10 \mathrm{ml}$ of PBS. We isolated CD4+ T cells using anti-CD4+ microbeads by column separation (Miltenyi Biotec, Auburn, CA), [10] using $20 \mathrm{ul}$ anti-CD4+ Micro beads per $10^{6}$ total cells. Pilot studies confirmed CD4+ cell yields of $\sim 4 \times$ $10^{6}$ at $\geq 95 \%$ purity per collection. Total RNA was extracted using the RNeasy Mini Protocol (QIAGEN, Valencia, CA) [11] and stored at $-80^{\circ}$ C. 2100 Bioanayzer (Agilent Technologies, Santa Clara, CA) analysis confirmed average total RNA yields of 2 ug per collection, with minimal evidence of RNA degradation and 28S:16S ratios approaching 2.0. Written informed consent was obtained from participants. CAMP was approved by the Institutional Review Boards of Brigham and Women's Hospital and the other participating centers.

\section{Microarray hybridization and sample preprocessing}

Expression profiles were generated with Illumina HumanRef8 v2 BeadChip arrays (Illumina, San Diego CA) using $100 \mathrm{ng}$ of CD4+ total RNA from each sample and the Illumina BeadStation 500G according to protocol [12]. Arrays were read using the BeadArray scanner (Illumina) and analyzed using BeadStudio (version 3.1.7) without background correction. Raw expression intensities were processed using the lumi package [13] with background adjustment with RMA convolution [14] and $\log _{2}$ transformation of each array. The combined set of arrays was subsequently quantile normalized. The Gene Expression Omnibus number for this dataset is GSE22324.

\section{Quantitative Real-Time PCR}

For validation of our microarray results using real-time PCR (RT-PCR) was performed on a subset of subjects ( $n=10$ ) with the Taqman 7900 gene expression assay using primers and probes from Applied Biosystems. Quantitative RT-PCR analyses were performed in triplicate with appropriate positive and negative controls. The mRNA values were normalized with TATA binding protein (TBP) serving as the internal control. Relative mRNA expression levels were calculated using the delta cycle time $(\mathrm{Ct})$ method.

\section{Serum Total IgE}

Total serum IgE was measured by radioimmunosorbent assays from blood samples collected during the CAMPCS/2 visits. Serum IgE was transformed to the $\log _{10}$ scale for analysis.

\section{Pulmonary Function and Methacholine challenge testing}

Pre- and post-bronchodilator spirometry was performed according to American Thoracic Society recommendations with a volume-displaced spirometer, and airway responsiveness was assessed by methacholine challenge with the Wright nebulizer tidal breathing technique [15].

\section{Statistical analysis}

All expression microarray analyses were performed in $\mathrm{R}$ 2.9/Bioconductor [16,17]. Variance-based non-specific filtering was used to remove uninformative probes specifying a minimum interquartile range of 1 on the $\log _{2}$ scale. We used the Limma package [18] to analyze the linear correlation between gene expression level and $\log _{10} \operatorname{IgE}$ measurements both unadjusted for covariates and adjusted for age, sex (where appropriate), ethnicity, and center of sample collection. Joint and sex-stratified analyses were performed to assess the influence of sex. To control for multiple testing, we used the FDR procedure as proposed by Benjamini and Hochberg [19] with a threshold of 0.05 to define statistical significance. To generate multivariate linear models accounting for the large- $p$ (expression transcript levels) small- $n$ (number of subjects) problem, and to accommodate correlations among predictor variables, we used the least absolute shrinkage and selection operator (LASSO) [20] estimation. LASSO is a coefficient shrinkage and variable selection method that achieves model sparsity by setting coefficients of many irrelevant transcripts to 0 . The LASSO analysis was performed using the least angle regression (LARS) algorithm, utilizing 5-fold cross-validation, as implemented in the $\mathrm{R}$ package lars. [21] We performed Canonical Pathway Analyses using Ingenuity Pathway Analysis ([IPA], Ingenuity Systems ${ }^{\circledR}$, http://www. ingenuity.com) software (utilizing gene transcripts attaining nominal levels of significance $[\mathrm{p}<0.05]$ in all subjects and within males and females specifically). 


\section{Results}

Expression data from peripheral blood CD4+ $\mathrm{T}$ cells was measured in 299 asthmatics from CAMP; 223 of these (75\%) also had a simultaneous measurement of total serum IgE. The baseline characteristics of the 223 CAMP subjects included in this analysis are presented in Table 1. There were no significant differences in any clinical variable between subjects with and without IgE measurement. We tested for evidence of linear association between gene transcript abundance in peripheral blood CD4+ T cells and total serum IgE. After non-specific gene filtering, 13,310 (64.5\%) transcripts demonstrating variable expression across samples were considered for testing.

\section{All Subjects}

\section{Single Gene Regressive Strategies}

Significant evidence for association between transcript abundance and total serum IgE was identified for a single gene, interleukin 17 receptor B (IL17RB), explaining
$12 \%$ of the variance $\left(\mathrm{r}^{2}\right)$ in total serum IgE measurement ( $\mathrm{p}$ value $=7 \times 10^{-7}, 9 \times 10^{-3}$ after adjustment for multiple testing, see Figure 1A). Similar results were noted in analyses restricted to the 168 white subjects (IL17RB, p value $=2 \times 10^{-6}, 2 \times 10^{-2}$ after adjustment for multiple testing), and technical validation by RT-PCR confirmed the association between total serum IgE levels and IL17RB transcript abundance (a positive correlation between delta $C T$ and total serum $\operatorname{IgE}\left[\mathrm{r}^{2} 0.37\right.$, p value $0.03])$. We re-analyzed our data with the inclusion of a quadratic term for gene expression level but found no significant findings of association under this non-linear modeling assumption. A list of the top 50 transcripts is presented in Additional File 1. No differences were noted in models additionally adjusting for measures of severity including methacholine $\mathrm{PC}_{20}$ (a measurement of airway responsiveness) or pre-bronchodilator $\mathrm{FEV}_{1}$ (a measurement of pulmonary function). Of note, exploratory analyses of the relationship between IL17RB transcript abundance and other intermediate asthma phenotypes revealed evidence of association with both

Table 1 Baseline characteristics of subjects from the Childhood Asthma Management Program (CAMP)

\begin{tabular}{|c|c|c|c|}
\hline \multirow[t]{2}{*}{ Variable } & \multicolumn{3}{|c|}{ Median (interquartile range) or Count (\%) } \\
\hline & All $(n=223)$ & Males $(n=134)$ & Females $(n=89)$ \\
\hline Age, years & $20(19-22)$ & $20(19-22)$ & $21(19-23)$ \\
\hline Sex, female & $89(40 \%)$ & - & - \\
\hline Ethnicity, white & $168(75 \%)$ & $97(72 \%)$ & $71(80 \%)$ \\
\hline African-American & $42(18 \%)$ & $26(19 \%)$ & $16(18 \%)$ \\
\hline Hispanic & $13(6 \%)$ & $11(8 \%)$ & $2(2 \%)$ \\
\hline Total serum IgE (IU/ml) & $356(145-924)$ & $480(150-1172)$ & $253(133-597)$ \\
\hline Atopy ${ }^{*} \dagger$ & $200(90 \%)$ & $122(91 \%)$ & $78(88 \%)$ \\
\hline \multicolumn{4}{|l|}{ Allergen Specific Skin Tests * $\neq$} \\
\hline \multicolumn{4}{|l|}{ Dust Mites } \\
\hline Dermatophagoides farinae & $97(44 \%)$ & $61(46 \%)$ & $36(40 \%)$ \\
\hline Dermatophagoides pternohyssinus & $92(41 \%)$ & $58(43 \%)$ & $34(38 \%)$ \\
\hline \multicolumn{4}{|l|}{ Cockroaches } \\
\hline Blattella germanica & $66(30 \%)$ & $41(31 \%)$ & $25(28 \%)$ \\
\hline Periplaneta Americana & $56(25 \%)$ & $36(27 \%)$ & $20(22 \%)$ \\
\hline Atopic dermatitis ${ }^{*} \neq \S$ & $48(22 \%)$ & $72(43 \%)$ & $15(31 \%)$ \\
\hline Allergic rhinitis ${ }^{*} \neq \S$ & $92(42 \%)$ & $56(43 \%)$ & $33(35 \%)$ \\
\hline FEV1 (liters) $\neq$ & $3.65(3.13-4.19)$ & $4.04(3.65-4.48)$ & $3.13(2.86-4.48)$ \\
\hline$\overline{F E V 1 / F V C ~(\%) ~} \neq$ & $78(72-83)$ & $76(71-82)$ & $79(75-84)$ \\
\hline PC20 $(\mathrm{mg} / \mathrm{ml}) \neq$ & $2.49(1.64-4.88)$ & $3.01(1.88-4.88)$ & $1.95(1.37-4.53)$ \\
\hline Used Inhaled Corticosteroids $\neq \|$ & $50(25 \%)$ & $24(21 \%)$ & $26(31 \%)$ \\
\hline Used Oral Prednisone $\neq \|$ & $12(6 \%)$ & $1(1 \%)$ & $11(13 \%)$ \\
\hline
\end{tabular}

* Data on these atopy variables was recorded at enrollment into the CAMP trial ( 10-12 years prior to both the gene expression and total serum IgE measurement).

† Atopy defined as subjects with at least one positive allergen skin test.

\# Data missing for skin test positive responses to Blattella Germanica in 1 subject, atopic dermatitis FEV1 and FEV1/FVC ratio in 9 subjects, for inhaled corticosteroids or oral prednisone in 23 subjects, and PC20 in 34 subjects.

$\S$ Atopic dermatitis and allergic rhinitis were defined by physician diagnosis.

|| Refers to subjects who used these medications within 7 days of both the gene expression and total serum lgE measurement. 

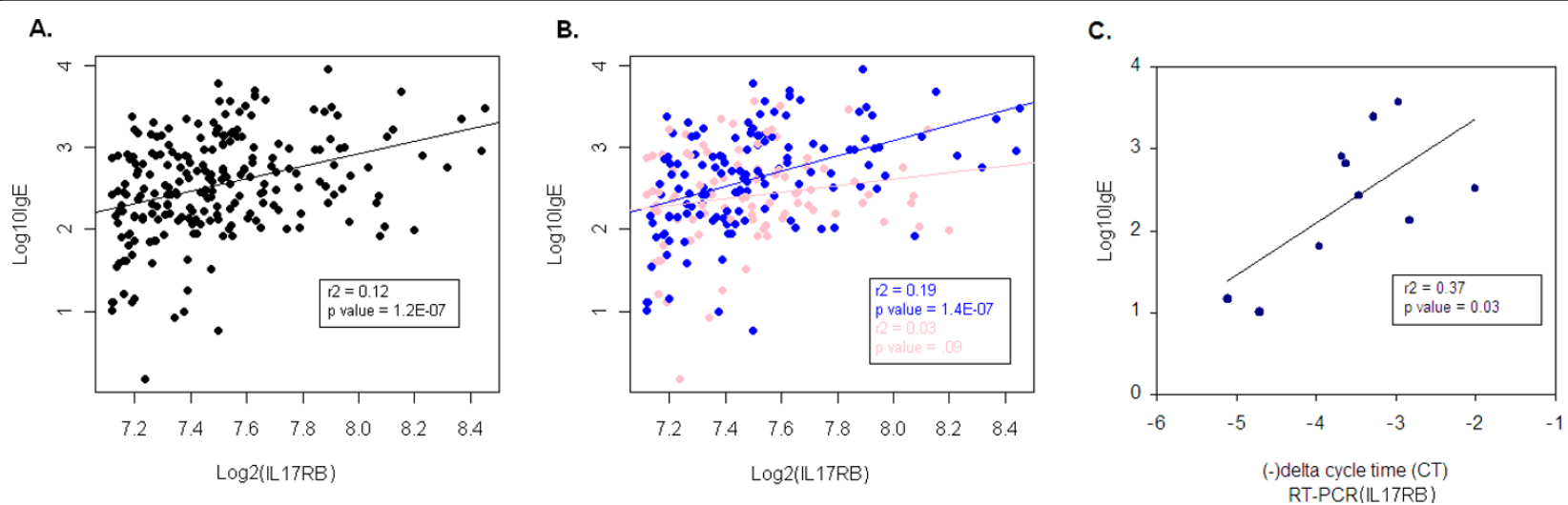

Figure 1 The Correlation Between CD4+ Lymphocyte Measurement of IL17RB and Serum IgE. Figure $1 \mathrm{~A}$ and 1B represent plots of log $2^{-}$ transformed gene expression measurement of IL17RB (on the $x$-axis) vs. $\log _{10}$-transformed total serum IgE measurement (on the $y$-axis), with regression lines, for all subjects without $(1 \mathrm{~A})$ and with (1B) consideration of male (in blue) and female (pink) sex. Figure $1 \mathrm{C}$ represents a plot of the (-) delta cycle time from the real time-polymerase chain reaction of measurement of IL17RB (on the $x$-axis) vs. $\log _{10}$-transformed total serum IgE measurement (on the $y$-axis) in all subjects.

total serum eosinophil count $\left(\mathrm{p}\right.$ value $\left.=6 \times 10^{-6}\right)$ and methacholine $\mathrm{PC}_{20}\left(\mathrm{p}\right.$ value $\left.=4 \times 10^{-4}\right)$.

Due to the well-documented sexual dimorphism of IgE levels, we assessed whether sex would modify the association between gene expression and total serum IgE by repeating the analysis for males $(\mathrm{n}=134,60 \%)$ and females $(\mathrm{n}=89,40 \%)$ separately. These analyses revealed that the correlation between IL17RB and total serum IgE was restricted to males only $\left(\mathrm{r}^{2}=0.19\right.$, $\mathrm{p}$ value $=8 \times 10^{-8}, 1 \times 10^{-3}$ after adjustment for multiple testing), and was not observed in females $\left(\mathrm{r}^{2}=.03, \mathrm{p}\right.$ value $=0.13,1$ after adjustment for multiple testing). Formal testing confirmed the observed differences in correlation of IL17RB and IgE in males and females was statistically significant (test for sex-specific interaction $\mathrm{p}$ $<0.05$, see Figure 1B). It is important to note that the differences in correlation between IL17RB and IgE were not due to baseline differences in IL17RB levels between males and females (mean $\log _{2}$ IL17RB level 7.5 in both males and females, $t$-test $\mathrm{p}$ value 0.5 for the difference). No other transcript was associated with total serum IgE among either males or females after correction for multiple testing. Plots of the linear correlation between IL17RB level and total serum IgE in males and females specifically, are presented in Figure 1B. The gene transcript with the strongest correlation with total serum IgE among CAMP females (gamma-aminobutyric B receptor 1 [GABBR1], unadjusted p-value $9 \times 10^{-4}$ ) did not remain significant after adjustment for multiple testing $(\mathrm{p}=0.9998)$. Lists of the top 50 transcripts for both males and females are presented in Additional Files 2, 3.

Next we examined the degree of overlap in the correlations between gene transcript measurement and total serum IgE between males and females. At an arbitrary cutoff ( $\mathrm{p}$ value $<0.05$ ), there were 872 and 154 transcripts demonstrating correlation with total serum IgE among males and females, respectively. Only 16 of these 1010 unique transcripts (1.6\%) were significantly correlated with total serum IgE in both males and females; and this result falls within the 95\% confidence interval ([95\% CI $]$ 5-17) of the number of transcripts that should overlap by chance alone. At a more liberal threshold for inclusion of the results from females $(\mathrm{p}<0.1)$, only 38 of 1205 unique transcripts (3.1\%) overlap. At a more conservative threshold $(\mathrm{p}<0.01)$ there were no transcripts common to both males and females.

We considered whether our findings of association in males but not females could be attributable to differences in sample size. Though power was somewhat lower in females due to the smaller number of subjects (89 females vs. 139 males), power was very high (>91\%) to detect correlations half as strong as those observed among males (power $91.4 \%$ to detect an $\mathrm{r}^{2}$ of 0.09 in the 89 CAMP females).

\section{LASSO}

Next we evaluated whether an alternate multi-gene modeling approach utilizing 5 -fold cross validation (the LASSO method) could both confirm our findings of association from single gene regressive strategies, and could identify additional determinants of total serum IgE levels. Among all subjects, the optimal multivariate model included 17 transcripts (including IL17RB), of which 16 map to unique HUGO gene identifiers (see Additional File 4). Consistent with our single gene regressive strategy the best fitting model includes 
$I L 17 R B$, the transcript with the largest estimated regression coefficient. Among males, the optimal multivariate model included 30 transcripts, including 6 that overlap with the best fitting regression model for all subjects: ASB9, H2A histone family, member J (H2AFJ), hexose6-phosphate dehydrogenase (H6PD), IL17RB, poly(A) binding protein interacting protein 1 (PAIP1), and PVALB (see Additional File 5). Again, IL17RB was the transcript with the largest estimated regression coefficient among males. For females, LASSO selected the null model as its best prediction model, which suggests that none of the genes were informative in predicting IgE level.

\section{Ingenuity Canonical Pathway Analysis}

To better understand the combinatorial effect of multiple gene transcripts on IgE regulation we performed canonical pathway analyses. Of the 763 transcripts demonstrating nominally significant $(\mathrm{p}<0.05)$ correlation with total serum IgE, 652 (85\%) map to a unique HUGO gene id and were used for IPA canonical pathway analysis. Among all subjects, enrichment for six pathways was observed (Fisher's exact $\mathrm{p}<0.05$ ) including the antigen presentation pathway, propanoate metabolism, interferon signaling, primary immunodeficiency signaling, the coagulation system, and the pantothenate and CoA biosynthesis pathway (see Figure 2A). Similar to the regression models, sex-stratified pathway analysis revealed different patterns of pathway enrichment between males and females. Among males, nominal evidence for enrichment was observed for 13 pathways (Figure 2B). Among females, enrichment was observed for 6 pathways (Figure 2C), including strong evidence of enrichment for the interferon signaling pathway in females $\left(p=3.0 \times 10^{-6}\right)$. Notably, there were no significant canonical pathways in common between males and females. Additional information about the canonical pathway analyses, including lists of differentially expressed genes within the pathways are included in Additional Files 6, 7, 8. Of note, IL17RB is not currently a constituent of an Ingenuity canonical pathway.

\section{Discussion}

This is the first study to correlate genome-wide CD4+ T cell transcript abundance with IgE level in humans and is, to our knowledge, the largest gene-expression study of as asthma-related quantitative phenotype published to date. Our results indicate that IL17RB may be the only gene expressed in CD4+ T cells whose transcript measurement is correlated with the variation in IgE level in asthmatics. Given that IgE level is strongly influenced by environmental factors (such as allergen exposures) it is surprising that $12 \%$ of the variation in $\operatorname{IgE}$ can be explained by IL17RB transcript measurement, a correlation that is substantially greater than that explained genetic determinants from three significant genomic regions $\left(\mathrm{r}^{2}=1.9 \%\right)$ identified in a recent genome-wide association study of IgE [22].

While it is often not possible within the confines of a cross-sectional epidemiologic study to determine the cause and effect relationship between two correlated variables, abundant molecular biologic evidence suggests that elevation of IL17RB should occur upstream from increases in IgE. IL17RB, located on chromosome 3p21.1, is the receptor for interleukin 17 B (IL17B) but has much higher binding avidity to interleukin $17 \mathrm{E}$ ([IL17E] also known as IL25, IL-17Rh1, and Evi27 in the mouse) [23]. While structural homology places the IL17E-IL17RB ligand-receptor pathway within the IL17 family, as opposed to IL17A and IL17F which are cytokines characteristic of Th17 cells, [24] several groups have demonstrated that the IL17E-IL17RB pathway is a strong inducer of Th2 responses [25]. In humans, IL17RB expression has been demonstrated on the surface of lung fibroblasts, [26] prominently on activated CD4+ Th2 memory cells, [27] and recently in basophils [28]. Comparable to our findings, IL17RB has recently been shown to be the most differentially expressed gene in allergen challenged peripheral blood mononuclear cells from patients seasonal allergic rhinitis compared to healthy controls [28]. Thymic stromal lymphopoietin activated DCs appear to provide an important activating signal allowing IL17RB + Th2 memory cells to respond to IL17E [27]. Elevated IL17E expression results in elevated Th2 cytokine production, [29] asthma severity, and elevations in IgE [30].

Another finding of our analyses is that the magnitude of the relationship between patterns of CD4+ lymphocyte gene expression and total serum IgE levels is substantially different between males and females. Several lines of evidence are provided, including: strong association of IL17RB transcript abundance with IgE levels in males $\left(r^{2}=19 \%\right)$, but not in females $\left(r^{2}=3 \%\right)$; evidence for a multivariate gene signature of total IgE levels in males, but not in females; and discrete, non-overlapping canonical gene pathway enrichment in each sex. Notably, we found limited statistical evidence to suggest that there is significant overlap between $\mathrm{CD} 4+\mathrm{T}$ cell genes whose transcript measurements correlate with IgE level in both male and female asthmatics. Our work stands with a body of literature now implicating an important role for sex in the heritability, genome-wide linkage analyses, [7] and genome-wide gene expression analyses (this report) of IgE, and strongly support the notion that genetic and genomic studies of IgE should consider the role of sexual dimorphism. 


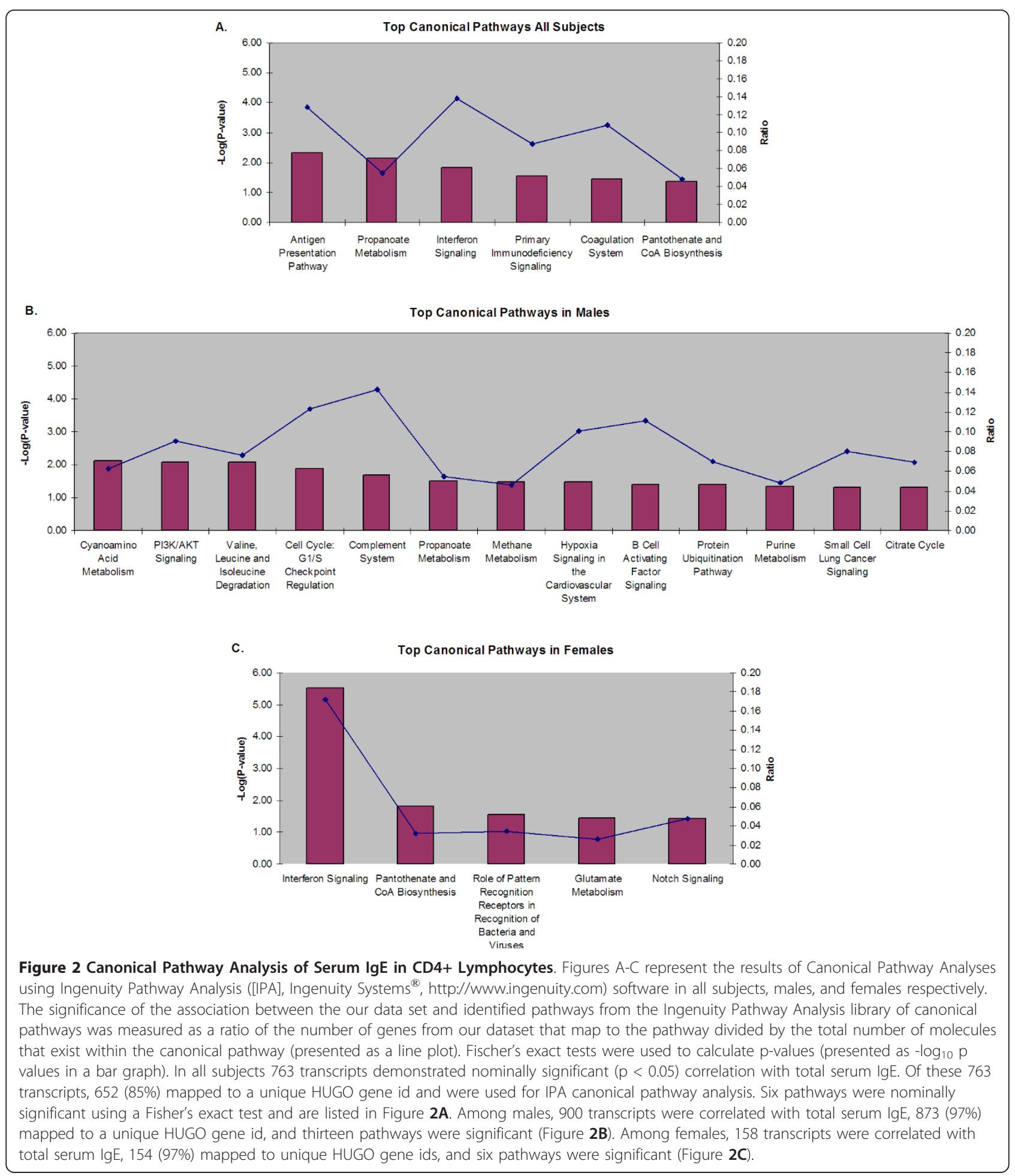

Our study has several limitations. While our study is among the largest gene expression studies of asthma and allergy to date it is important to note that sample size may still be an important limitation of our analyses. The imbalance in sample size between males and females in this study, and the inherently lower mean values of IgE levels in females can further reduce statistical power. Though our power calculations suggest ample power $(>90 \%)$ to detect the male-specific associations in our female sample, replication in larger sample sizes could help to determine if the lack of overlap we note between males and females is primarily an issue of the magnitude 
of the correlations or of true differences in the genomic regulation of IgE. In addition, our gene expression analyses include gene transcript measurements from unstimulated peripheral blood CD4+ lymphocytes in a population of adolescents and young adults recruited on the basis of childhood asthma. We urge caution in extrapolating our findings to other populations and cell types. Finally, while an assessment of IL17RB protein levels would provide additional supportive information, we do not have intact $\mathrm{CD} 4+$ lymphocytes enabling suitable cell surface protein quantification.

While the precise role that sex plays in the genomic regulation of IgE is unknown, sex differences in the immune environments experienced by $\mathrm{CD} 4+\mathrm{T}$ cells are well described. In animals, females are more likely to develop a Th1 response after antigen challenge, except during pregnancy when a Th2 environment dominates [31]. Although little is known about the role sex could play in IL17RB pathway regulation, some evidence suggests that IL17RB level may influence the response to sex hormones [32]. We recently demonstrated sex-specific effects of TSLP polymorphisms on the regulation of specific, and total serum IgE in children with asthma [33]. This is intriguing given that TSLP activated dendritic cells (TSLP DCs) appear to be one of the most potent stimuli of IL17RB expression [27].

Though we have limited evidence that single genes, or genetic models strongly correlate with IgE level among females, it is intriguing that the canonical pathway most significantly correlated with IgE in females was the interferon signaling pathway $\left(\mathrm{p}=3 \times 10^{-6}\right)$, particularly in light of prior studies suggesting the importance of interferon- $\gamma$ (INFG) in the female (more than male) allergic response [34]. In mice, females demonstrate increased T-cell proliferative responses, [34] and consistently produce increased INF $\gamma$ [35] in response to antigen challenge compared with their male counterparts. In humans, females have been shown to increase INF $\gamma$ levels in response to a variety of stimuli [36]. Thus, our observation of differential down-regulation of the IFN signaling in females (but not in males) with higher levels of IgE is consistent with these prior studies and suggests an important regulatory role for interferon signaling in the female allergic response.

\section{Conclusions}

Our results indicate that IL17RB may be the only gene expressed in CD4+ T cells whose transcript measurement is correlated with the variation in IgE level in asthmatics. We have demonstrated that sex is a critical modifier of the correlation between the CD4+ T cell transcriptome and IgE, and suggest that such sex-specific modification should be consideration in future studies of IgE.

\section{Additional material}

Additional File 1: Top 50 genes whose expression is correlated with total serum $\mathrm{lgE}$ in all subjects.

Additional File 2: Top 50 genes whose expression is correlated with total serum IgE in males.

Additional File 3: Top 50 genes whose expression is correlated with total serum $\lg E$ in females.

Additional File 4: Genes included in Lasso model for all subjects.

Additional File 5: Genes included in Lasso model for males. Additional File 3a:

Additional File 6: Canonical pathways (and genes) correlated with total serum IgE in all subjects.

Additional File 7: Canonical pathways (and genes) correlated with total serum $\lg \mathrm{E}$ in males.

Additional File 8: Canonical pathways (and genes) correlated with total serum IgE in females.

\section{Acknowledgements}

We thank all subjects for their ongoing participation in this study. We acknowledge the CAMP investigators and research team, supported by $\mathrm{NHLB}$, for collection of CAMP Genetic Ancillary Study data. Special thanks to Teresa Concordia, Debbie Bull, Anne Plunkett, Denise Rodgers, and D. Sundstrom for their assistance with sample collection. We thank Drs. Andy Liu and Stanley Szefler (National Jewish, Denver CO), Nadia Hansel, Greg Diette and N. Franklin Adkinson Jr. (Johns Hopkins, Baltimore MD), and Karen DeMuth, Robert Strunk and Mario Castro (Washington University, St. Louis MO), Anne Fuhlbrigge (Brigham and Women's Hospital, Boston MA) for their assistance in collection of CD4+ lymphocytes and RNA extraction. We thank Chris Allaire, Barbara Klanderman, and Stephanie Metje for their assistance with sample collection and processing. All work on data collected from the CAMP Genetic Ancillary Study was conducted at the Channing Laboratory of the Brigham and Women's Hospital and Harvard Medical School under appropriate CAMP policies and human subject's protections.

All work on data collected from the CAMP Genetic Ancillary Study was conducted at the Channing Laboratory of the Brigham and Women's Hospital under appropriate CAMP policies and human subject protections. This work is supported by National Heart, Lung and Blood Institute, National Institutes of Health (NIH/NHLBI) grant R01 HL086601. The CAMP Genetics Ancillary Study is supported by U01 HL075419, U01 HL65899, P01 HL083069, N01 HR16049, and T32 HL07427 from the NIH/NHLBI. G.M.H. is supported by K08 HL092222. M.H.C., S.S.S., and A.J.R. are supported by K12 HL089990 from the NHLBI. B.E.H. is supported by 2T15LM007092-16 from the National Library of Medicine.

This paper is subject to the NIH public access policy: http://www.nih.gov/ about/publicaccess/Finalpublicaccessimplementation031505.htm.

\section{Author details}

${ }^{1}$ Channing Laboratory, Brigham and Women's Hospital, Harvard Medical School, Boston, MA 02115, USA. ${ }^{2}$ Division of Pulmonary and Critical Care Medicine, Brigham and Women's Hospital, Boston, MA 02115, USA. ${ }^{3}$ Center for Genomic Medicine, Brigham and Women's Hospital, Boston, MA 02115, USA. ${ }^{4}$ Harvard-MIT Division of Health Sciences and Technology, Cambridge, MA, USA.

\section{Authors' contributions}

GMH conducted the majority of the statistical analyses and drafted the manuscript. JHC, SSS, MHC, BEH, AJR, AM, participated in statistical analyses, and contributed to drafting this manuscript. VJC helped to create and modify the statistical packages used in these analyses, and contributed to drafting this manuscript. BAR conceived of the study, and participated in its design and coordination and helped to draft the manuscript. All authors read and approved the final manuscript.

\section{Competing interests}

Dr. Raby reports receiving lecture fees from Novartis Pharmaceuticals. All of the other authors report no conflict of interest. 
Received: 29 October 2010 Accepted: 7 April 2011

Published: 7 April 2011

\section{References}

1. Burrows B, Martinez FD, Halonen M, Barbee RA, Cline MG: Association of asthma with serum IgE levels and skin-test reactivity to allergens. N Engl J Med 1989, 320:271-277.

2. Sears MR, Burrows B, Flannery EM, Herbison GP, Hewitt CJ, Holdaway MD: Relation between airway responsiveness and serum IgE in children with asthma and in apparently normal children. N Engl J Med 1991, 325:1067-1071.

3. Geha RS, Jabara HH, Brodeur SR: The regulation of immunoglobulin $E$ class-switch recombination. Nat Rev Immunol 2003, 3:721-732.

4. Guilbert TW, Morgan WJ, Zeiger RS, Bacharier LB, Boehmer SJ, Krawiec M, Larsen G, Lemanske RF, Liu A, Mauger DT, et al: Atopic characteristics of children with recurrent wheezing at high risk for the development of childhood asthma. J Allergy Clin Immunol 2004, 114:1282-1287.

5. Uekert SJ, Akan G, Evans MD, Li Z, Roberg K, Tisler C, Dasilva D, Anderson E, Gangnon R, Allen DB, et al: Sex-related differences in immune development and the expression of atopy in early childhood. J Allergy Clin Immunol 2006, 118:1375-1381.

6. Kerkhof M, Droste JH, de Monchy JG, Schouten JP, Rijcken B: Distribution of total serum IgE and specific lgE to common aeroallergens by sex and age, and their relationship to each other in a random sample of the Dutch general population aged 20-70 years. Dutch ECRHS Group, European Community Respiratory Health Study. Allergy 1996, 51:770-776.

7. Raby BA, Soto-Quiros ME, Avila L, Lake SL, Murphy A, Liang C, Fournier E, Spesny M, Sylvia JS, Verner A, et al: Sex-specific linkage to total serum immunoglobulin $\mathrm{E}$ in families of children with asthma in Costa Rica. Hum Mol Genet 2007, 16:243-253.

8. Weiss $L A$, Pan $L$, Abney M, Ober $C$ : The sex-specific genetic architecture of quantitative traits in humans. Nat Genet 2006, 38:218-222.

9. The Childhood Asthma Management Program (CAMP): design, rationale, and methods. Childhood Asthma Management Program Research Group. Control Clin Trials 1999, 20:91-120.

10. Jonuleit $H$, Schmitt $E$, Schuler $G$, Knop J, Enk AH: Induction of interleukin 10-producing, nonproliferating $\mathrm{CD} 4(+) \mathrm{T}$ cells with regulatory properties by repetitive stimulation with allogeneic immature human dendritic cells. J Exp Med 2000, 192:1213-1222.

11. Gonzalez P, Zigler JS Jr, Epstein DL, Borras T: Identification and isolation of differentially expressed genes from very small tissue samples. Biotechniques 1999, 26:884-886, 888-892.

12. Barnes M, Freudenberg J, Thompson S, Aronow B, Pavlidis P: Experimental comparison and cross-validation of the Affymetrix and Illumina gene expression analysis platforms. Nucleic Acids Res 2005, 33:5914-5923.

13. Du P, Kibbe WA, Lin SM: lumi: a pipeline for processing Illumina microarray. Bioinformatics 2008, 24:1547-1548.

14. Irizarry RA, Hobbs B, Collin F, Beazer-Barclay YD, Antonellis KJ, Scherf $U$, Speed TP: Exploration, normalization, and summaries of high density oligonucleotide array probe level data. Biostatistics 2003, 4:249-264.

15. Smyth GK: Bioinformatics and Computational Biology Solutions using $R$ and Bioconductor New York: Springer; 2005.

16. R Development Core Team: R: A Language and Environment for Statistical Computing. Vienna, Austria; 2009.

17. Gentleman RC, Carey VJ, Bates DM, Bolstad B, Dettling M, Dudoit S, Ellis B, Gautier L, Ge Y, Gentry J, et al: Bioconductor: open software development for computational biology and bioinformatics. Genome Biol 2004, 5:R80.

18. Smyth GK: Linear models and empirical bayes methods for assessing differential expression in microarray experiments. Stat Appl Genet Mol Biol 2004, 3, Article3.

19. Benjamini Y, Drai D, Elmer G, Kafkafi N, Golani I: Controlling the false discovery rate in behavior genetics research. Behav Brain Res 2001, 125:279-284.

20. Tibshirani R: Regression shrinkage and selection via the lasso. Journal of the Royal Statistical Society Series B 1995.

21. Tibshirani R: The lasso method for variable selection in the Cox model. Stat Med 1997, 16:385-395.

22. Weidinger $S$, Gieger $C$, Rodriguez E, Baurecht $H$, Mempel M, Klopp N, Gohlke H, Wagenpfeil S, Ollert M, Ring J, et al: Genome-wide scan on total serum IgE levels identifies FCER1A as novel susceptibility locus. PLOS Genet 2008, 4:e1000166.
23. Tian E, Sawyer JR, Largaespada DA, Jenkins NA, Copeland NG, Shaughnessy JD Jr: Evi27 encodes a novel membrane protein with homology to the IL17 receptor. Oncogene 2000, 19:2098-2109.

24. Wang YH, Liu YJ: The IL-17 cytokine family and their role in allergic inflammation. Curr Opin Immunol 2008, 20:697-702.

25. Angkasekwinai P, Park H, Wang YH, Chang SH, Corry DB, Liu YJ, Zhu Z, Dong C: Interleukin 25 promotes the initiation of proallergic type 2 responses. J Exp Med 2007, 204:1509-1517.

26. Lajoie-Kadoch S, Joubert P, Letuve S, Halayko AJ, Martin JG, SoussiGounni A, Hamid Q: TNF-alpha and IFN-gamma inversely modulate expression of the IL-17E receptor in airway smooth muscle cells. Am J Physiol Lung Cell Mol Physiol 2006, 290:L1238-1246.

27. Wang YH, Angkasekwinai P, Lu N, Voo KS, Arima K, Hanabuchi S, Hippe A, Corrigan CJ, Dong C, Homey B, et al: IL-25 augments type 2 immune responses by enhancing the expansion and functions of TSLP-DCactivated Th2 memory cells. J Exp Med 2007, 204:1837-1847.

28. Wang H, Mobini R, Fang Y, Barrenas F, Zhang H, Xiang Z, Benson M: Allergen challenge of peripheral blood mononuclear cells from patients with seasonal allergic rhinitis increases IL-17RB, which regulates basophil apoptosis and degranulation. Clin Exp Allergy 40:1194-1202.

29. Fort MM, Cheung J, Yen D, Li J, Zurawski SM, Lo S, Menon S, Clifford T, Hunte B, Lesley R, et al: IL-25 induces IL-4, IL-5, and IL-13 and Th2associated pathologies in vivo. Immunity 2001, 15:985-995.

30. Ballantyne SJ, Barlow JL, Jolin HE, Nath P, Williams AS, Chung KF, Sturton G, Wong SH, McKenzie AN: Blocking IL-25 prevents airway hyperresponsiveness in allergic asthma. J Allergy Clin Immunol 2007, 120:1324-1331.

31. Whitacre CC, Reingold SC, O'Looney PA: A gender gap in autoimmunity. Science 1999, 283:1277-1278.

32. Jerevall PL, Brommesson $S$, Strand C, Gruvberger-Saal S, Malmstrom P, Nordenskjold B, Wingren S, Soderkvist P, Ferno M, Stal O: Exploring the two-gene ratio in breast cancer-independent roles for HOXB13 and IL17BR in prediction of clinical outcome. Breast Cancer Res Treat 2008, 107:225-234.

33. Hunninghake GM, Lasky-Su J, Soto-Quiros ME, Avila L, Liang C, Lake SL, Hudson TJ, Spesny M, Fournier E, Sylvia JS, et al: Sex-stratified linkage analysis identifies a female-specific locus for IgE to cockroach in Costa Ricans. Am J Respir Crit Care Med 2008, 177:830-836.

34. Weinstein $Y$, Ran $S$, Segal $S$ : Sex-associated differences in the regulation of immune responses controlled by the MHC of the mouse. I Immunol 1984, 132:656-661

35. Gourdy P, Araujo LM, Zhu R, Garmy-Susini B, Diem S, Laurell H, Leite-deMoraes M, Dy M, Arnal JF, Bayard F, Herbelin A: Relevance of sexual dimorphism to regulatory T cells: estradiol promotes IFN-gamma production by invariant natural killer T cells. Blood 2005, 105:2415-2420.

36. Hewagama A, Patel D, Yarlagadda S, Strickland FM, Richardson BC: Stronger inflammatory/cytotoxic T-cell response in women identified by microarray analysis. Genes Immun 2009, 10:509-516.

\section{Pre-publication history}

The pre-publication history for this paper can be accessed here: http://www.biomedcentral.com/1471-2466/11/17/prepub

doi:10.1186/1471-2466-11-17

Cite this article as: Hunninghake et al:: The CD4+ T-cell transcriptome and serum IgE in asthma: IL17RB and the role of sex. BMC Pulmonary Medicine 2011 11:17. 